\title{
HACIA LA INUTILIDAD: \\ Una crítica a la dependencia digital
}

\author{
Alba Aragón Estrada \\ Universidad de Sevilla
}

\begin{abstract}
Resumen: Hoy en día todos contamos con un inseparable compañero en nuestros bolsillos, el teléfono "inteligente", una ventana a un mundo sin límites, Internet. Esto ha propiciado que muchas de nuestras actividades se hayan subcontratado en diversos aparatos electrónicos y en la misma Red, lo que me lleva a preguntarme si nos estamos volviendo excesivamente dependientes de dichas tecnologías. Estudios recientes demuestran que, mediante el uso excesivo de Internet, estamos privilegiando cada vez más las rutas neuronales encargadas de un procesamiento rápido y superficial de la información en detrimento de aquellas dedicadas al pensamiento profundo y el razonamiento. Debido a ello, puede surgir la pregunta de si nos estamos volviendo inútiles a la hora de ejecutar dicho pensamiento y por ende de filosofar al modo que hemos venido haciendo hasta ahora.
\end{abstract}

Palabras clave: atención, autonomía epistémica, (in)dependencia epistémica, integración cognitiva, inutilidad, mejora cognitiva, memoria, pensamiento profundo, procesamiento de la información, rutas neuronales, tecnologías intelectuales.

Toward uselessness: a review of digital dependency

Abstract: Nowadays everyone relies on an inseparable partner in our pockets, a Smartphone, the window to an endless world, the Internet. This has contributed to many of our activities to be outsourced in various electronic devices and in the Network, which makes us question ourselves if we are becoming excessively depending on such technologies. Recent studies have proved that an excessive use of the Internet reinforces even more certain neural routes in charge of a faster and shallow information processing, in detriment of those routes dedicated to deep thought and reasoning. This leads us to wonder if we are becoming unfit when it comes to executing said thought and therefore to philosophise the way we have been doing it until now. 
Keywords: attention, cognitive enhancement, cognitive integration, data processing, deep thinking, epistemic (in)dependence, epistemic self-direction, intellectual technologies, memory, neural routes, uselessness.

Recibido: 07/10/2018 Aprobado: 20/02/2019

\section{Introducción}

La tecnología ha formado parte del desarrollo del ser humano desde los albores de la humanidad. Como diría Martin Heidegger (Heidegger, 1994), nos enfrentamos al mundo con aquello que tenemos a-la-mano; hasta tal punto, que se ha demostrado que el desarrollo del cerebro va parejo de la utilización de determinadas "herramientas", desde piedras hasta libros y nuestras últimas adquisiciones, los teléfonos "inteligentes". Así pues, la humanidad ha llegado a ser lo que es gracias a los útiles que ha empleado durante el proceso de hominización y posterior enculturación.

No obstante, una amenaza se cierne sobre nuestras mentes (y cerebros); el uso excesivo de las nuevas tecnologías digitales. Su desarrollo ha tenido durante las últimas décadas un auge que no conoce precedentes, llegando a extenderse hasta los más mínimos resquicios de la sociedad. En nuestra vida diaria utilizamos aparatos electrónicos para absolutamente todo, desde leer hasta orientarnos por ciudades desconocidas; hasta tal punto, que se puede considerar que el que una vez fue homo faber y luego homo sapiens se está convirtiendo ahora en homo technologicus.

Este nuevo contexto de mejora de las capacidades cognitivas mediante la utilización de aparatos tecnológicos ha hecho surgir dudas sobre cómo afecta su uso a nuestra capacidad epistémica: cestamos delegando demasiado la formación de nuestras creencias acerca del mundo (y nuestro sistema de conocimiento) en estos aparatos?, ¿nos estamos volviendo dependientes de estos aparatos a la hora de llevar a cabo procesos de razonamiento?, ¿̇iensan ellos por nosotros?, ¿nos queda autonomía epistémica o la hemos donado toda a estos aparatos? ¿Supone su uso una mejora de las capacidades cognitivas sin producir fallas? Intentaré contestar estas preguntas a lo largo del presente ensayo. 


\section{La tecno-batalla}

En los últimos años se han planteado diversos puntos de discusión referentes al papel que las tecnologías digitales tienen en nuestra mejora cognitiva y sobre todo en relación con nuestra (in)dependencia epistémica. Este debate tiene dos vertientes bien definidas: los tecnófobos y los tecnófilos.

Por un lado, se hallan los que consideran que el progreso tecnológico y el posthumanismo han abocado a una "anti-mejora" basándose sobre todo en consideraciones éticas que tildan al mejoramiento cognitivo como peligroso e inmoral. Figuras como Michael Patrick Lynch (Lynch, 2016) o Nicholas Carr (Carr, 2014) defienden que este tipo de mejoras mediante el uso de tecnologías puede llegar a minar nuestra capacidad para formar adecuadamente razonamientos. Como consecuencia, se perjudicaría a la formación del pensamiento y reflexión profundos, provocando que se pierda aquello que nos aporta dignidad como humanos. Así pues, Lynch (Lynch, 2016) llega a plantear que estamos llegando a un punto en el que la tecnología sustituye la capacidad de pensar por nosotros mismos hasta tal extremo que, si llegara a ocurrir un desastre natural, nos dejaría en un muy mal lugar. Por su parte Nicholas Carr (Carr, 2014) defiende que vivimos en un estado de «complacencia automatizada», es decir, «confiamos que las máquinas resolverán todo de tal manera que nos encomendamos a ellas como si fueran todopoderosas» $\mathrm{y}$ «evitando que desarrollemos talentos que solo se pueden lograr cuando se lucha duro por conseguir las cosas». A la par, hay quienes argumentan como Persson y Savulescu (Persson y Savulescu, 2012) que la mejora cognitiva es demasiado peligrosa, ya que las tecnologías disponibles han llevado a que los seres humanos cometan las mayores atrocidades de su historia. Estas posiciones representan un movimiento llamado tecnofobia, el miedo a que la tecnología nos deje indefensos, dentro de los cuales encontramos también bioconservadores como Lynch y Carr, que sostienen que la excesiva dependencia en estos aparatos acaba por socavar las capacidades biológicas para la conformación de la epistemología. 
Por otra parte, se encuentran los tecnófilos, quienes defienden que el uso de las nuevas tecnologías nos lleva sin duda a una mejora cognitiva sin causar perjuicio ninguno. Clive Thompson manifiesta que: «casi he dejado de esforzarme en recordar cualquier cosa porque cualquier información se puede recabar online al instante» y además «mediante la descarga de datos en silicio, liberamos nuestra propia materia gris para tareas más relevantemente humanas, como dejar volar las ideas y soñar despierto» (Thompson, 2007). Por su parte, Peter Sudermann (Sudermann, 2009) defiende que las conexiones a Internet, al estar continuamente disponibles, hacen que no sea eficiente usar el cerebro como almacén de información y por ello es mejor almacenarla digitalmente y solo recordar que la hemos almacenado. Don Tapscott sostiene esta misma opinión: «Ahora que podemos consultar cualquier cosa con un clic en Google, memorizar largos pasajes o hechos históricos se ha quedado obsoleto. La memorización es una pérdida de tiempo.» (Tapscott, 2009:115). Otro ejemplo de cómo la tecnología nos ayuda a la mejora cognitiva lo encontramos dentro de la concepción de la mente extendida con autores como Sutton (Sutton, 2010) y Heersmink (Heersmink, 2015a , Heersmink, 2015b). Su postura consiste en defender el andamiaje cognitivo aportado por las nuevas tecnologías como una clase de mejora de los procesos cognitivos biológicos.

Dentro de esta concepción es interesante la perspectiva de J. Adam Carter, quien defiende que algunas clases de mejoras cognitivas son, de hecho, constitutivas de la virtud de la autonomía intelectual. Sostiene que, dado que nuestras facultades cognitivas biológicas fallan a veces, la confianza en la tecnología es del todo pertinente siempre y cuando (siguiendo a Goldman, 1976) la mejora cognitiva se integre de manera correcta en el sistema cognitivo. O lo que es lo mismo, sin producir desajustes en el sujeto que le impidan funcionar como un todo. La clave del argumento de Carter y Goldman está pues en la integración cognitiva.

Este será el concepto a partir del cual desarrollaré el presente ensayo, en el que intentaré defender que la excesiva dependencia de las tecnologías digitales produce desajustes en la capacidad cognitiva a la hora de proceder 
a dicha integración. Esto podría volvernos de alguna manera "inútiles" para el pensamiento profundo, base de la correcta ejecución de una epistemología bien fundamentada.

Para ello examinaremos en primer lugar el modo en que se ha constituido nuestra capacidad de razonamiento a partir del uso de diversas tecnologías a lo largo de la historia. A continuación, expondré cómo dicho uso afecta a la neuroplasticidad del cerebro y, en concreto, a la capacidad de la memoria. Finalmente, concluiré que nuestra forma tradicional de filosofar puede estar tocando a su fin, debido a que las capacidades de atención y memoria (fundamentales en los procesos de formación de pensamiento profundo, base de la reflexión filosófica) están siendo debilitadas por las tecnologías digitales.

\section{Tecnologías intelectuales}

Las tecnologías intelectuales son aquellas herramientas que utilizamos para ampliar o apoyar nuestra capacidad mental, encontrar y clasificar la información, formular y articular ideas, compartir métodos y conocimientos, tomar medidas y realizar cálculos, y ampliar la capacidad de nuestra memoria (Cfr. Carr, 2011: 62). Dentro de esta denominación encontramos ejemplos como la máquina de escribir, el ábaco, el libro, la escuela, y por supuesto los ordenadores e Internet.

Desde hace tiempo ha quedado patentemente demostrado que el uso de dichas tecnologías ejercen un poder grande y duradero sobre qué y cómo pensamos, hasta tal punto que llegan incluso a conformar nuestra identidad personal $1^{1}$. Los útiles que usamos para escribir, leer y manipular la información «trabajan nuestra mente tanto como nosotros trabajamos con ellas»

\footnotetext{
${ }^{1}$ Así pues, vemos como la introducción del uso del mapa impulsó el pensamiento abstracto configurando a la mente y haciéndola apta para entender el entorno. Del mismo modo, el reloj supuso que nuestro trabajo mental estuviera primado por la división del tiempo y con ello la búsqueda de las estructuras que componen las unidades complejas.
} 
Alba Aragón

(Carr, 2011: 66) pues su uso fortalece determinados circuitos neuronales mientras que debilita otros.

Es de vital importancia analizar cómo el uso de este tipo de tecnologías ha afectado a la mente y con ella a nuestro cerebro ${ }^{2}$. Y para ello el primer paso es analizar cómo afectan a nuestro lenguaje, puesto que es el «vaso del pensamiento consciente» (Carr, 2011). Esto nos lleva a considerar cómo la forma de su trasmisión y ejecución le afecta a sí mismo. Se sabe por numerosos estudios que la lectura y la escritura, bajo la educación y la práctica, fomentan una conformación deliberada del cerebro. ${ }^{3}$ Esto sugiere que el modo de escribir y hablar influye directamente en el pensamiento de tal manera que un cambio en éstos producirá que pensemos de manera diferente. Un ejemplo ilustrativo lo proporciona el filósofo Friedrich Nietzsche, quien, tras comenzar a escribir a máquina a partir del aumento de su ceguera, escribe a un amigo: «Tenéis razón. Nuestros útiles de escritura participan en la formación de nuestros pensamientos» (Cit. en Carr, 2011, 32).

Siguiendo la premisa del socavamiento de la integración cognitiva por parte de las nuevas tecnologías, la forma que tenemos de escribir y leer en las redes nos está llevando a ejecutar un pensamiento superficial que produce alteraciones rápidas y poco profundas en los circuitos neuronales. ¿Por qué? Porque proporciona tal multitud de estímulos que no podemos discriminar la información verdaderamente importante de la que no lo es, debido a que nos abruma, lo que produce un procesamiento de la información muy rápido que no permea. Dado que repetimos en multitud de ocasiones este proceso de lectura rápida y superficial, las rutas neuronales que lo soportan son las que están siendo consolidadas.

${ }^{2}$ Ha quedado demostrado que la influencia que ejerce el medio en nuestra actividad mental configura el cerebro de tal manera que provoca cambios fisiológicos y anatómicos en su estructura.

${ }^{3}$ Parece muy interesante a este respecto como Peter Sloterdijk argumenta en su obra Normas para el parque humano que la cultura humanista basada en la transmisión (a la manera de relación epistolar) de obras escritas ha supuesto el adoctrinamiento de los humanos, en la cual ha tenido un papel preponderante la escuela. 
En el siguiente apartado pasaré pues a examinar cómo la neuroplasticidad del cerebro conduce a que estos tipos de procesamientos sean los que queden "guardados" como rutas rápidas de acceso mientras que se olvidan los utilizados para la lectura atenta y profunda, propios del pensamiento filosófico que conocemos desde el siglo IV. a.C.

\section{Cerebros de plastilina}

La base fundamental de los argumentos del apartado anterior y, que a su vez conforman los cimientos de mi hipótesis, es la neuroplasticidad. Según esta tesis, largamente puesta en duda durante el siglo XX, pero posteriormente confirmada, la estructura del cerebro está siempre en constante flujo, adaptándose a las tareas que se le encomienda incluso en la madurez ${ }^{4}$. Esto significa que las células del cerebro se desarrollan y aumentan de tamaño con el uso y se atrofian o consumen por falta del mismo (Cfr. Young, 1951: 36). O en palabras de William James:

Las aguas que fluyen abren un cauce por el que fluir, lo van haciendo más ancho y profundo; y cuando vuelve a fluir, seguirán el mismo camino que trazaron antes. Asimismo, las impresiones de los objetos externos van diseñando caminos cada vez más apropiados en el sistema nervioso; y estos caminos vitales se reabren con similar estimulación externa, incluso aunque hayan estado cortados algún tiempo (Cit. en Carr, 2011: 35).

En otras palabras, cada vez que realizamos una tarea o experimentamos una sensación se activan un conjunto de neuronas. Al repetirse la misma experiencia los enlaces sinápticos se hacen progresivamente más fuertes y abundantes, dado que van produciendo cambios fisiológicos y anatómicos en el cerebro. Esto hará que se afiancen más ciertas rutas cerebrales, y, por

\footnotetext{
${ }^{4}$ Aunque es cierto que con el paso de los años el cerebro va perdiendo dicha plasticidad, no obstante, no desaparece del todo.
} 
consiguiente, actuemos de manera más automática. Poco a poco se tenderá a crear "adicción" por las viejas rutas ${ }^{5}$, siendo muy difícil abandonarlas y crear unas nuevas.

Dicha plasticidad, condición de posibilidad de la evolución, es un arma de doble filo, pues «si dejamos de ejercer nuestra capacidad mental el cerebro no se limita a olvidar: el espacio que dedicaba a las viejas habilidades se entrega a las nuevas habilidades que se practican en su lugar» (Doidge, 2007: 59).

He aquí entonces la clave que andaba buscando; el excesivo uso de Internet en la vida diaria fomenta una distracción constante mediante la cacofonía de estímulos que en él impera. Asimismo, cortocircuita el pensamiento tanto consciente como inconsciente y entorpece que la mente pueda pensar de forma profunda y creativa. Dado que pasamos un tiempo "infinito" gozando de sus virtudes, su uso provocará alteraciones fundamentales en nuestra disposición mental, que pueden llegar a perpetuarse ocupando el lugar que antes se disponía para la lectura tranquila y profunda, base de una integración cognitiva correcta.

Veremos a continuación el porqué de esta tajante afirmación.

\section{La muerte de Mnemosine}

La atención y la memoria figuran entre las capacidades cognitivas más importantes para la formación de un sistema epistémico sin fallas. Por la primera se produce la entrada de "estímulos" en nuestra mente-cerebro y por la segunda las creencias que han sido elaboradas a partir de éstos pasan estar a nuestra disposición para lograr conformar pieza a pieza un sólido edificio epistemológico. En este apartado vamos a analizar cómo afecta el uso continuo de la Red a estas dos capacidades tan fundamentales y cómo, si no le ponemos remedio, acabaremos siendo "ineptos epistémicos".

\footnotetext{
${ }^{5}$ Ciertamente el proceso por el que se aprende a tocar un instrumento musical viene a ser este; trazar una ruta neuronal y recorrerla una y otra vez hasta hacerla automática. 
La memoria a largo plazo constituye la sede del entendimiento pues no sólo almacena hechos, sino también conceptos complejos y esquemas. Asimismo, permite organizar datos dispersos bajo un patrón de conocimiento y capacita para desarrollar un pensamiento profundo (Cfr. Carr, 2011: 153). Por otro lado, la memoria de trabajo permite un almacenamiento sólo temporal de la información, de manera que ésta permanecerá muy poco tiempo en el cerebro. No obstante, su importancia es vital puesto que proporciona a la memoria a largo plazo el material con el que habrá de trabajar luego. Por consiguiente, si se produjera un problema en la memoria de trabajo como, por ejemplo, la saturación por una insistencia continuada de estímulos, la memoria a largo plazo no podría funcionar adecuadamente y la información no se integraría de forma correcta.

La dispersión de la atención y la solución de problemas superfluos, principales tareas que se ejecutan cuando se está en internet, provocan dicha sobrecarga cognitiva. Un ejemplo sería el hipertexto: cuando estamos leyendo un artículo en internet nos asaltan enlaces que nos redirigen a temas relacionados. Seguramente la curiosidad nos incitará a pinchar en ellos e investigar más, pues somos consumidores natos de información. Al repetir este proceso una y otra vez, al final, resultará que nos hemos distraído de la lectura del artículo principal y llegamos a olvidar el tema tratado. Como esta lectura online se hace de una manera muy superficial (rara vez posamos más de diez segundos nuestra vista sobre una misma página web), el aporte a la memoria de trabajo no queda lo suficientemente afianzado. Resulta irónico pues que, con tanta información como ofrece Internet, el vagabundeo de una página a otra lo único que consigue es diluir la atención y con ello una pobre adquisición de conocimiento.

Conviene recordar en ese punto la referencia que hice al lenguaje al tratar el tema de las tecnologías intelectuales. Vimos que éste se ejecuta y desarrolla mediante la escritura y la lectura, de tal modo que si se produce algún cambio en éstas también cambiará el lenguaje, y con él, el pensamiento, ya que el primero es vehículo del segundo. Ahora bien, resulta que la lectura y la comprensión exigen el establecimiento de relaciones entre 
conceptos, hacer inferencias, activar conocimientos previos (almacenados en la memoria a largo plazo gracias a una integración correcta de la información que nos provee la memoria de trabajo) y sintetizar ideas. Si se produce pues una desorientación en la carga de la memoria de trabajo, ésta puede interferir con las actividades cognitivas de comprensión (Cfr. Carr, 2011: 160). Así, la división de la atención que exigen las tecnologías digitales sobrecarga las capacidades cognitivas haciendo que disminuya el aprendizaje y debilitando el entendimiento. Carr dice que «cuando se trata de aportar a la mente la materia de la que están hechos los pensamientos, puede que más sea menos» (Carr, 2011: 160).

Todo ello implica que este desplazamiento constante de la atención producirá un cerebro ágil a la hora de realizar múltiples tareas. El mejoramiento de esta capacidad conlleva a que otras, como la de pensar profunda y creativamente se vean socavadas, de tal manera que, a más multitarea, menor capacidad para pensar y razonar un problema. En palabras de Lynch:

Puede ser algo bueno; pero también puede debilitar y socavar otras formas de conocimiento, formas que requieren una captación más holística y creativa de cómo la información se encuentra conectada (Lynch, 2016: 6).

Como consecuencia, las rutas neuronales que propician este tipo de pensamiento serán abandonadas en beneficio de aquellas que posibilitan saltar rápidamente de una tarea a otra, con el consiguiente déficit de atención. Y lo que resulta más peligroso, los hábitos que adquirimos en la red también funcionan cuando no estamos conectados a ella. ${ }^{6}$

${ }^{6}$ Es interesante advertir que al principio de su libro Carr reseña cómo poco a poco, mediante el uso de herramientas de procesamiento de texto, ha ido convirtiéndose él mismo en un procesador de textos. Como hemos visto, el modo en que escribimos, leemos y hablamos va configurando la forma de pensar. Como experiencia personal, recuerdo cuando empecé también a utilizar este tipo de herramientas, sobre todo las de presentación mediante diapositivas. Mi mente empezó a dejarse permear por el método esquemático que utilizaba 
Esto supondrá que poco a poco iremos entrenando la mente-cerebro para que se entregue a funciones que ayudan a localizar, clasificar y evaluar rápidamente fragmentos de información dispares. ${ }^{7}$ Lo que significa que, al no estar orientados hacia una comprensión profunda y personalmente construida de los textos, la red disminuye la capacidad de conocer profundamente una materia por nosotros mismos y construir un pensamiento profundo y rico (Cfr. Carr 2011: 175-176).

La conclusión de este apartado es ineludible: debido a que recibimos multitud de mensajes cuando estamos conectados a Internet, se produce una sobrecarga en nuestra memoria de trabajo, haciendo más difícil que podamos concentrar la atención. Si esta primera fase falla, el proceso de consolidación de la memoria no puede siquiera empezar. Dada la plasticidad de las redes neuronales, cuanto más usemos la Red más nos entrenamos para la distracción y el procesamiento de información rápido y carencia de atención. A medio y largo plazo se irá haciendo más difícil el almacenamiento de información en la memoria biológica, viéndonos entonces obligados a depender cada vez más de la memoria artificial de la Red y a otras prótesis de almacenamiento, haciendo que nos volvamos más superficiales ${ }^{8}$ como pensadores. (Cfr. Carr, 2011).

\section{Hacia la inutilidad}

Llegamos pues al final de nuestro viaje y por tanto a la hora de las conclusiones.

para diseñar las diapositivas, hasta el punto de llegar a cambiar el modo en el que establecía los esquemas sobre las lecciones que estudiaba en mi cuaderno de escritura a mano.

${ }^{7}$ Como también reseña Carr, este tipo de funciones son muy similares a las realizadas por los ordenadores, programados para la trasferencia a alta velocidad de datos. Esto significa que estamos adoptando en nosotros mismos las características de la tecnología. (Carr, 2011, 175)

${ }^{8}$ El termino superficial utilizado aquí por Carr incluye una de las facetas que pretendemos que se englobe dentro del término que da título a este ensayo, inutilidad. 
La primera de ellas es que, dado que las capacidades para el procesamiento profundo de información se basan en la adquisición consciente de conocimiento, el análisis inductivo, el pensamiento crítico, la imaginación y la reflexión, y dado que el mundo de la Red exige una atención dispersa, la toma de decisiones sin apenas reflexionar (algunas de ellas incluso inconscientes), además de suponer una sobrecarga de estímulos, produce que el uso continuado de la Red entorpezca la formación de un sistema epistémico firme.

En segundo lugar, la atención, primer eslabón de la cadena de integración cognitiva, se muestra dispersa sin poder aportar datos a la memoria de trabajo debido a las exigencias de multitareas que exige la conexión a la Red. Por tanto, el uso de ésta provoca que no pueda desarrollarse adecuadamente la formación de creencias acerca del mundo en que hemos de desenvolvernos.

En tercer lugar, dado que la continua afluencia de datos y la dispersión de la atención cuando estamos online produce una sobrecarga de la memoria de trabajo que colapsa el proceso de traspaso de dicha información a la memoria a largo plazo, el uso continuo de las tecnologías asociadas a las redes socava el proceso de integración cognitiva.

De todo ello se deriva el siguiente "corolario": dado que Internet desintegra la capacidad cognitiva de la memoria, su uso continuado provoca que la integración cognitiva no se produzca de manera correcta, lo cual es un argumento contra el uso de las tecnologías asociadas a la red.

En quinto lugar, gracias a la neuroplasticidad del cerebro, las experiencias físicas y mentales que experimentamos habitualmente van trazando rutas que se afianzan mediante repetición. Debido a que hoy en día estamos continuamente ejecutando las rutas asociadas a la gestión de información online, éstas tomarán un lugar preponderante, haciendo que se abandonen las destinadas al pensamiento profundo.

Por último, concluyo que Internet nos está configurando para un entorno que no es aquel en el que tenemos que desarrollarnos diariamente. Ello es debido a que la experiencia con el medio configura el cerebro produciendo 
modificaciones fisiológicas y anatómicas mediante el trazado de rutas cada vez más transitadas que lo hacen apto para desarrollarse en su entorno. Sin embargo, Internet está produciendo el trazado de rutas adecuadas a su entorno siendo este distinto (aunque no en todo punto) del entorno donde nuestra vida usual se desarrolla (como diría Heidegger el mundo, el $d a$ del Dasein). Y dada de nuevo la neuroplasticidad del cerebro que fija rutas neuronales mediante hábitos repetidos, podría suceder que, si fallara la conexión a Internet o los aparatos a ella asociado, no supiéramos desarrollarnos en un entorno para el que ya no tenemos pautas neuronales. Aunque bien visto, la propia neuroplasticidad sería la condición de posibilidad para nuevas adaptaciones al nuevo entorno.

En resumen, mi posición recae del lado de los que en el primer apartado denominé bioconservadores pues, al igual que opina Carr, sostengo que se está cometiendo una falacia al equiparar nuestros procedimientos y capacidades cognitivas con la de los ordenadores y demás aparatos electrónicos. Me parece que se olvida mediante esta metáfora que, si bien estos procesamientos se parecen, los humanos somos más, somos seres biológicos que cuentan con una memoria biológica que ha supuesto la base de la propia constitución del conocimiento científico y filosófico.

Finalmente, como defensora de la cognición extendida, me gustaría sostener una postura híbrida en la que el uso de las tecnologías digitales sea acompañado también por las tecnologías analógicas, de tal manera que las rutas neuronales asociadas tanto a unas como a otras se mantengan activas. Si el dicho popular dice «en la variedad está el gusto» propongo «en la variedad de procesos cognitivos para la constitución de una epistemología firme está el conocimiento.» Así pues, defiendo el uso de las tecnologías digitales para la mejora cognitiva pero no el abuso y siempre mostrándonos cautos hacia las supuestas ventajas que el uso de dichas tecnologías pueda tener. 
Alba Aragón

\section{Bibliografía empleada}

N. Carr, Superficiales: ¿Quue está haciendo internet con nuestras mentes?, Madrid, Taurus, 2011.

— "Hacia el "homo technologicus", en: El País, 2014, https://elpais.com/sociedad/ 2014/09/19/actualidad/1411146383_037635.html. Accedido el 13 de febrero de 2018.

J. A. Carter, "Intellectual autonomy, epistemic dependence and cognitive enhancement", en: Synthese, 2017: 1-25.

N. Doidge, El cerebro se cambia a sí mismo, Madrid, Aguilar, 2008.

A. Goldman, "Discrimination and perceptual knowledge", en: Journal of Philosophy, 1976, 73(20): 771-791.

R. Heersmink, (a) "Extended mind and cognitive enhancement: Moral aspects of cognitive artifacts", en: Phenomenology and the Cognitive Sciences, 2015: 17-32.

-, (b) "Dimensions of integration in embedded and extended cognitive systems", en: Phenomenology and the Cognitive Sciences, 2015, 14(3): 577-598.

M. Heidegger, "La pregunta por la técnica", en: Conferencias y artículos, Traducción de Eustaquio Barjau, Barcelona, Serbal, 1994.

M. P. Lynch, The Internet of us: Knowing more and understanding less in the age of big data, London, W.W. Norton, 2016.

I. Persson, y J. Savulescu, Unfit for the future: The need for moral enhancement. Oxford: OUP, Oxford, 2012.

P. Sloterdijk, Normas para el parque humano: Una respuesta a la carta sobre el humanismo de Heidegger, traducción de Teresa Rocha Barco, Madrid, Siruela, 2000.

P. Suderman, "Your Brain Is an Index", en: American Scene, 2009 www.theamericnscene.com/2009/05/11/your-braiin-is-an-index . Accedido 9 febrero 2018

J. Sutton, "Exograms and interdisciplinarity: History, the extended mind, and the civilizing process", 2010, en: R. Menary. (Ed.), The extended mind, Cambridge: MIT Press, 2013: 189-225.

D. Tapscott, Grown Up Digital, Nueva York, McGraw-Hill, 2009.

C, Thompson, "Your Outboard Brain", en: The New York Times, 2007 Accedido 10 febrero 2018.

J. Z. Young, Doubt and certainty in science: A Biologist's on the Brain, Londres, Oxford University Press, 1951.

Alba Aragón Estrada

albaraest@alum.us.es 